\title{
Ensuring Secrecy in MIMO Wiretap Channels With Imperfect CSIT: A Beamforming Approach
}

\author{
Amitav Mukherjee and A. Lee Swindlehurst \\ Electrical Engineering and Computer Science \\ University of California \\ Irvine, CA 92697-2625 \\ a.mukherjee@uci.edu; swindle@uci.edu
}

\begin{abstract}
In this paper, we investigate transmit beamforming schemes based on artificial interference for physical layer security in a multi-antenna wiretap channel. We consider the case where no information regarding the eavesdropper is present, and we use the signal-to-interference-plus-noise-ratio of a single transmitted data stream as our performance metric. Using a second-order perturbation analysis, we quantify the degradation in performance due to imperfect CSI of the main channel at the transmitter. We then present a robust beamforming scheme that approaches the performance obtained in the perfect CSI case. Numerical simulations verify our analytical performance predictions, and illustrate the benefits of the robust beamforming scheme.
\end{abstract}

\section{INTRODUCTION}

There has recently been considerable interest in the use of physical layer mechanisms to increase the security of wireless communications links. An analytical scenario involving a legitimate transmitter-receiver pair and a passive eavesdropper is commonly referred to as the wiretap channel [1]-[3]. The primary information-theoretic measure of performance in such channels is the secrecy capacity, which quantifies the rate at which a transmitter can reliably send a secret message to the receiver, without the eavesdropper being able to decode it. Recent work has extended the secrecy capacity metric to the multiple-antenna (MIMO) wiretap channel, where all nodes may possess multiple antennas [4]-[7]. Broadly speaking, a non-zero secrecy capacity can only be obtained if the eavesdropper's channel is less capable than that of the intended recipient.

However, to compute the secrecy rate, one must know the eavesdropper's channel state information (CSI), or at least its distribution. Such information is unlikely to be available in many scenarios, especially those involving purely passive eavesdroppers. As a result, in this paper we take a different approach in which the transmitter allocates to the desired receiver only those resources necessary to guarantee a certain Quality of Service (QoS), and uses the remaining resources to transmit an artificial interference signal that jams any eavesdroppers that are present [8], [9]. The use of artificial interference has been considered by a number of others even for the case where the eavesdropper's CSI is known [10],

This work was supported by the U.S. Army Research Office under the Multi-University Research Initiative (MURI) grant W911NF-07-1-0318. although such an approach is known to be suboptimal. The high-SNR performance of this type of technique was shown to be nearly optimal in [4], and the optimal power distribution between data and interference has been examined in [11]. Intentional interference with multiple cooperating receivers has been considered in [12].

Another key consideration is the accuracy of the CSI available at the transmitter. The impact of imperfect CSI on the secrecy rate of the single-antenna wiretap channel has been investigated in [13], [14]. As we illustrate, techniques based on knowledge of the eavesdropper's channel are very sensitive to even slight perturbations in the CSI. Imprecise CSI for the primary channel can also cause interference leakage to the desired recipient when artificial noise is used to jam the eavesdropper, resulting in significant degradation in the desired user's performance.

We consider a situation in which beamforming is used at the transmitter for the desired signal rather than spatial multiplexing, due to the increased robustness of beamforming in general, and to make the problem tractable. We adopt received signal-to-interference-plus-noise-ratio (SINR) as our performance metric, and we derive robust beamforming schemes at both the transmitter and receiver to minimize the effect of the imprecise CSI. Our results are based on a second-order perturbation analysis of the primary channel's singular value decomposition, and rely on knowledge of the variance of the CSI perturbation.

\section{SYSTEM MODEL WITH PERFECT CSI}

\section{A. Mathematical Model}

We assume a 3-user scenario where Alice and Bob constitute the legitimate transmitter-receiver pair, and a passive eavesdropper Eve who attempts to listen in to ongoing transmissions. Each of the nodes may possess multiple antennas, the number of which we denote by $N_{a}, N_{b}$ and $N_{e}$, respectively. The signals received by Bob and Eve can be represented as follows:

$$
\begin{aligned}
& \mathbf{y}_{b}=\mathbf{H}_{b a} \mathbf{x}_{a}+\mathbf{n}_{b} \\
& \mathbf{y}_{e}=\mathbf{H}_{e a} \mathbf{x}_{a}+\mathbf{n}_{e},
\end{aligned}
$$

where $\mathbf{x}_{a}$ is the signal vector transmitted by Alice, $\mathbf{n}_{b}, \mathbf{n}_{e}$ are the naturally occurring noise and interference received by Bob 
and Eve, respectively, and $\mathbf{H}_{b a}, \mathbf{H}_{e a}$ are the corresponding $N_{b} \times N_{a}, N_{e} \times N_{a}$ channel matrices. The channels $\mathbf{H}_{b a}, \mathbf{H}_{e a}$ are deterministic, and no assumptions are made about their size or structure.

The background noise is assumed to be spatially white, with possibly different power levels:

$$
E\left\{\mathbf{n}_{b} \mathbf{n}_{b}^{H}\right\}=\sigma_{b}^{2} \mathbf{I} \quad E\left\{\mathbf{n}_{e} \mathbf{n}_{e}^{H}\right\}=\sigma_{e}^{2} \mathbf{I},
$$

where $E\{\cdot\}$ denotes expectation, $(\cdot)^{H}$ the Hermitian transpose, and $\mathbf{I}$ is an identity matrix of appropriate dimension. The transmit power available for Alice is bounded by $P$ :

$$
E\left\{\mathbf{x}_{a} \mathbf{x}_{a}^{H}\right\}=\mathbf{Q}_{a} \quad \operatorname{Tr}\left(\mathbf{Q}_{a}\right) \leq P,
$$

where $\operatorname{Tr}(\cdot)$ denotes the trace operator.

\section{B. Artificial Interference}

A fraction of Alice's power is devoted to the transmission of a noise-like interference signal designed to degrade the ability of Eve to intercept the signal destined for Bob. Since we are focusing on a beamforming scenario, Alice's signal is split into two components: one being a scalar data stream denoted as $z$ that contains the message for Bob, and one that contains the jamming signal, which we denote by the $N_{a} \times 1$ vector $\mathbf{z}^{\prime}$. Bob therefore receives

$$
\mathbf{y}_{b}=\mathbf{H}_{b a} \mathbf{t} z+\mathbf{H}_{b a} \mathbf{z}^{\prime}+\mathbf{n}_{b},
$$

where $\mathbf{t}$ is the $N \times 1$ transmit beamformer used for the information signal. Similarly, Eve sees

$$
\mathbf{y}_{e}=\mathbf{H}_{e a} \mathbf{t} z+\mathbf{H}_{e a} \mathbf{z}^{\prime}+\mathbf{n}_{e} .
$$

Assume $\mathbf{t}^{H} \mathbf{t}=1$ and let $E\left\{|z|^{2}\right\}=\rho P$, where $0<\rho \leq 1$ is the fraction of the power devoted to the information signal, so that

$$
E\left\{\mathbf{z}^{\prime} \mathbf{z}^{H}\right\}=\mathbf{Q}_{z}^{\prime} \quad \operatorname{Tr}\left(\mathbf{Q}_{z}^{\prime}\right)=(1-\rho) P .
$$

The QoS experienced by Bob and the probability of Eve intercepting the message intended for Bob will be determined by Alice's choice of the following parameters: the covariance matrix $\mathbf{Q}_{z}^{\prime}$, the transmit beamformer $\mathbf{t}$, and the power allocation parameter $\rho$. The impact of these parameters on secrecy capacity and SINR are discussed below.

Without any information about $\mathbf{H}_{e a}$ whatsoever, maximization of the channel secrecy rate is an ill-posed problem. However, since we are restricting attention to the transmission of a single data stream between Alice and Bob, we choose to work directly with SINR. Let $\mathbf{w}_{b}, \mathbf{w}_{e}$ respectively denote the $N_{b} \times 1, N_{e} \times 1$ beamformers employed by Bob and Eve to determine $z$, so that

$$
\begin{aligned}
& \hat{z}_{b}=\mathbf{w}_{b}^{H} \mathbf{y}_{b}=\mathbf{w}_{b}^{H}\left(\mathbf{H}_{b a} \mathbf{t} z+\mathbf{H}_{b a} \mathbf{z}^{\prime}+\mathbf{n}_{b}\right) \\
& \hat{z}_{e}=\mathbf{w}_{e}^{H} \mathbf{y}_{e}=\mathbf{w}_{e}^{H}\left(\mathbf{H}_{e a} \mathbf{t} z+\mathbf{H}_{e a} \mathbf{z}^{\prime}+\mathbf{n}_{e}\right) .
\end{aligned}
$$

The resulting SINR available for Bob and Eve to decode $z$ will be given by

$$
\begin{aligned}
\operatorname{SINR}_{b} & =\frac{\rho P\left|\mathbf{w}_{b}^{H} \mathbf{H}_{b a} \mathbf{t}\right|^{2}}{\mathbf{w}_{b}^{H}\left(\mathbf{H}_{b a} \mathbf{Q}_{z}^{\prime} \mathbf{H}_{b a}^{H}+\sigma_{b}^{2} \mathbf{I}\right) \mathbf{w}_{b}} \\
\operatorname{SINR}_{e} & =\frac{\rho P\left|\mathbf{w}_{e}^{H} \mathbf{H}_{e a} \mathbf{t}\right|^{2}}{\mathbf{w}_{e}^{H}\left(\mathbf{H}_{e a} \mathbf{Q}_{z}^{\prime} \mathbf{H}_{e a}^{H}+\sigma_{e}^{2} \mathbf{I}\right) \mathbf{w}_{e}} .
\end{aligned}
$$

To illustrate the proposed artificial interference concept, we assume here that the CSI is perfectly known by all parties, Alice, Bob and Eve. The case where Bob and Alice have imperfect or perturbed CSI is examined in Section III.

The proposed approach can be generally outlined as follows:

1) Specify a target SINR for Bob.

2) Allocate a fraction $\rho$ of the available transmit power to achieve the desired SINR (if possible) assuming Bob experiences no interference other than the background noise of power $\sigma_{b}^{2}$.

3) Distribute Alice's remaining power to yield as much interference as possible, while guaranteeing that when the interference is received by Bob, it lies in a subspace orthogonal to the desired signal.

Let $S$ denote the target SINR for Bob. To minimize the fraction of the transmit power required to achieve $S$, Alice should choose $\mathbf{t}$ to be the right singular vector of $\mathbf{H}_{b a}$ with largest singular value, and Bob should choose $\mathbf{w}_{b}=\mathbf{H}_{b a} \mathbf{t}$ as his receive beamformer. Using this approach, we have

$$
\rho=\frac{\sigma_{b}^{2} S}{\mathbf{t}^{H} \mathbf{H}_{b a}^{H} \mathbf{H}_{b a} \mathbf{t} P}=\frac{\sigma_{b}^{2} S}{\sigma_{1}^{2} P},
$$

where $\sigma_{1}$ is the largest singular value of $\mathbf{H}_{b a}$. As long as $\rho<1$, Alice has power available for generating artificial interference.

Since the CSI of the eavesdropper is unknown, the best option available to Alice is to uniformly spread the remaining transmit power along spatial dimensions that will produce no interference for Bob. In particular, we require that

$$
\mathbf{H}_{b a} \mathbf{t} \perp \mathbf{H}_{b a} \mathbf{z}^{\prime}
$$

for all $\mathbf{z}^{\prime}$. With $\mathbf{t}$ chosen as above, it is easy to see that $\mathbf{z}^{\prime}$ must be chosen as a linear combination of the $N_{a}-1$ right singular vectors of $\mathbf{H}_{b a}$ with smallest singular values, which we denote by $\mathbf{T}^{\prime}$. Uniformly distributing the remaining transmit power over these vectors yields the following transmit covariance for the artificial interference:

$$
\mathbf{Q}_{z}^{\prime}=\frac{(1-\rho) P}{N_{a}-1} \mathbf{T}^{\prime} \mathbf{T}^{\prime H} .
$$

\section{IMPACT OF IMPERFECT CSI}

The assumption of perfect CSI at the transmitter is obviously impossible to achieve in practice. CSI uncertainty at Alice can be due to a number of different phenomena, including estimation error, quantized feedback, or channel mobility. CSI at the receiver is typically much more accurate, due to the receiver's ability to employ rapid channel tracking techniques based on, for example, decision direction. In this 
section, we examine the effect of inaccurate or mismatched CSI between Alice and Bob using a second-order perturbation analysis of the singular value decomposition (SVD) of $\mathbf{H}_{b a}$, assuming that the channel error is described as a zero-mean random matrix with known covariance.

Assume that $\mathbf{H}_{b a}$ is of full rank $F=\min \left(N_{b}, N_{a}\right)$, and define the singular value decomposition of the unperturbed channel as follows:

$$
\begin{aligned}
\mathbf{H}_{b a} & =\mathbf{U} \boldsymbol{\Sigma} \mathbf{V}^{H} \\
& =\left[\mathbf{U}_{s} \mathbf{u}_{F}\right]\left[\begin{array}{cc}
\boldsymbol{\Sigma}_{s} & 0 \\
0 & \sigma_{F}
\end{array}\right]\left[\mathbf{V}_{s} \mathbf{v}_{F}\right]^{H} \\
& =\mathbf{U}_{s} \boldsymbol{\Sigma}_{s} \mathbf{V}_{s}^{H}+\sigma_{F} \mathbf{u}_{F} \mathbf{v}_{F}^{H},
\end{aligned}
$$

where $\mathbf{U}_{s}, \mathbf{V}_{s}$ contain respectively the first $F-1$ left and right singular vectors whose singular values are found in the diagonal matrix $\boldsymbol{\Sigma}_{s}$, and $\mathbf{u}_{F}, \mathbf{v}_{F}$ are respectively the left and right singular vectors corresponding to the smallest singular value $\sigma_{F}$. The partitioning of the SVD will be useful as we use the perturbation analysis of [15].

For purposes of our analysis, we assume that the CSI error is confined to Alice, who is assumed to have available the following perturbed channel estimate:

$$
\tilde{\mathbf{H}}_{b a}=\mathbf{H}_{b a}+\Delta \mathbf{H}_{b a}
$$

where $\Delta \mathbf{H}_{b a}$ is modeled as a zero-mean circularly-symmetric random matrix with covariance given by

$$
\mathbf{C}_{\Delta \mathbf{H}_{b a}}=E\left\{\left(\operatorname{vec}\left(\Delta \mathbf{H}_{b a}\right)\right)\left(\operatorname{vec}\left(\Delta \mathbf{H}_{b a}\right)\right)^{H}\right\},
$$

and $\operatorname{vec}(\cdot)$ denotes the column stacking operator. In the sequel, we consider the case where the CSI errors are independent and identically distributed as $\mathbf{C}_{\Delta \mathbf{H}_{b a}}=\sigma_{H}^{2} \mathbf{I}$.

The singular value decomposition of the perturbed channel can be written as

$$
\tilde{\mathbf{H}}_{b a}=\tilde{\mathbf{U}}_{s} \tilde{\boldsymbol{\Sigma}}_{s} \tilde{\mathbf{V}}_{s}^{H}+\tilde{\sigma}_{F} \tilde{\mathbf{u}}_{F} \tilde{\mathbf{v}}_{F}^{H},
$$

where

$$
\begin{array}{ll}
\tilde{\mathbf{U}}_{s}=\mathbf{U}_{s}+\Delta \mathbf{U}_{s} & \tilde{\mathbf{u}}_{F}=\mathbf{u}_{F}+\Delta \mathbf{u}_{F} \\
\tilde{\boldsymbol{\Sigma}}_{s}=\boldsymbol{\Sigma}_{s}+\Delta \boldsymbol{\Sigma}_{s} & \tilde{\sigma}_{F}=\sigma_{F}+\Delta \sigma_{F} \\
\tilde{\mathbf{V}}_{s}=\mathbf{V}_{s}+\Delta \mathbf{V}_{s} & \tilde{\mathbf{v}}_{F}=\mathbf{v}_{F}+\Delta \mathbf{v}_{F} .
\end{array}
$$

We also define $\Delta \sigma_{1}$ and $\Delta \mathbf{v}_{1}$ as the perturbation to the largest singular value and the corresponding right singular vector $\mathbf{v}_{1}$, respectively. The analysis of [15] assumes a fat or square matrix ( $N_{a} \geq N_{b}$ in our case), so we perform our derivation for this case with the extension to $N_{b}>N_{a}$ being straightforward.

If Alice has an inaccurate estimate of the CSI and both Alice and Bob are unaware of the CSI mismatch, then the SINR for Bob is expected to be significantly degraded. This case of mismatched beamformers and erroneous power allocation due to imperfect CSI is referred to as the "naive" scheme.

We can also define the following second-order parameter perturbations:

$$
\Delta \sigma_{1} \approx\left[\Delta \boldsymbol{\Sigma}_{s}\right]_{1,1} \quad \Delta \sigma_{1}^{2} \approx\left[\Delta \boldsymbol{\Sigma}_{s} \Delta \boldsymbol{\Sigma}_{s}^{H}\right]_{1,1}
$$

$$
\begin{gathered}
\tilde{\sigma}_{1}^{2}=\sigma_{1}^{2}+2 \sigma_{1} \Delta \sigma_{1}+\Delta \sigma_{1}^{2} \\
\tilde{\rho}=\frac{\sigma_{b}^{2} S}{\tilde{\sigma}_{1}^{2} P} \approx \rho\left(1-\frac{2 \Delta \sigma_{1}}{\sigma_{1}}-\frac{\Delta \sigma_{1}^{2}}{\sigma_{1}^{2}}\right) .
\end{gathered}
$$

The average SINR achieved by Bob under the naive scheme is

$$
\operatorname{SINR}_{b}^{\text {naive }}=\frac{P E\left\{\tilde{\rho}\left|\mathbf{v}_{1}^{H} \mathbf{H}_{b a}^{H} \mathbf{H}_{b a}\left(\mathbf{v}_{1}+\Delta \mathbf{v}_{1}\right)\right|^{2}\right\}}{E\left\{\mathbf{v}_{1}^{H} \mathbf{H}_{b a}^{H}\left(\mathbf{H}_{b a} \tilde{\mathbf{Q}}_{z}^{\prime} \mathbf{H}_{b a}^{H}+\sigma_{b}^{2} \mathbf{I}\right) \mathbf{H}_{b a} \mathbf{v}_{1}\right\}},
$$

where the remaining expectation is with respect to $\Delta \mathbf{H}_{b a}$.

Based on the distribution of $\Delta \mathbf{H}_{b a}$, we can compute

$$
\begin{aligned}
E\left\{\mathbf{v}_{1}^{H} \mathbf{H}_{b a}^{H} \mathbf{H}_{b a} \tilde{\mathbf{Q}}_{z}^{\prime} \mathbf{H}_{b a}^{H} \mathbf{H}_{b a} \mathbf{v}_{1}\right\} & \left.=\sigma_{1}^{4} \tilde{\beta} E\left\{\mathbf{v}_{1}^{H}\left(\mathbf{I}-\tilde{\mathbf{v}}_{1} \tilde{\mathbf{v}}_{1}^{H}\right) \mathbf{v}_{1}\right\} 22\right) \\
& \approx-\sigma_{1}^{4} \beta E\left\{\mathbf{v}_{1}^{H} \Delta \mathbf{v}_{1}+\Delta \mathbf{v}_{1}^{H} \mathbf{v}_{1}\right\},
\end{aligned}
$$

where $\tilde{\beta}=(1-\tilde{\rho}) P /\left(N_{a}-1\right)$ and $\beta=(1-\rho) P /\left(N_{a}-1\right)$. Using the familiar relations $\mathbf{H}_{b a} \mathbf{v}_{1}=\sigma_{1} \mathbf{u}_{1}$ and $\mathbf{H}_{b a}^{H} \mathbf{u}_{1}=$ $\sigma_{1} \mathbf{v}_{1}$, after dropping higher-order perturbation terms from the numerator and denominator we obtain

$\operatorname{SINR}_{b}^{n}=\frac{\sigma_{1}^{2} \rho P\left[1+E\left\{\mathbf{v}_{1}^{H} \Delta \mathbf{v}_{1}\right\}+E\left\{\Delta \mathbf{v}_{1}^{H} \mathbf{v}_{1}\right\}-E\left\{\frac{2 \Delta \sigma_{1}}{\sigma_{1}}+\frac{\Delta \sigma_{1}^{2}}{\sigma_{1}^{2}}\right\}\right]}{-\sigma_{1}^{2} \beta\left[E\left\{\mathbf{v}_{1}^{H} \Delta \mathbf{v}_{1}\right\}+E\left\{\Delta \mathbf{v}_{1}^{H} \mathbf{v}_{1}\right\}\right]+\sigma_{b}^{2}}$

It is apparent that when perfect CSI is available at Alice (i.e., $\left.\sigma_{H}^{2}=0\right),(23)$ reduces to (9).

Next, we obtain the expected values of the perturbation terms $\mathbf{v}_{1}^{H} \Delta \mathbf{v}_{1}, \Delta \sigma_{1}$, and $\Delta \sigma_{1}^{2}$ in (23). Define

$$
\mathbf{D} \triangleq\left(\boldsymbol{\Sigma}_{s} \boldsymbol{\Sigma}_{s}^{H}-\sigma_{F}^{2} \mathbf{I}\right)^{-1}
$$

and

$$
\mathbf{E}_{i j} \triangleq \mathbf{U}_{i}^{H} \Delta \mathbf{H}_{b a} \mathbf{V}_{j}, i, j=s, n,
$$

as different projections of $\Delta \mathbf{H}_{b a}$. The perturbation in $\mathbf{V}_{s}$ can be approximated up to the second order of $\Delta \mathbf{H}_{b a}$ as [15]

$$
\Delta \mathbf{V}_{s}=\mathbf{v}_{F} \overline{\mathbf{P}}_{1}+\mathbf{V}_{s} \overline{\mathbf{P}}_{2}
$$

where $\overline{\mathbf{P}}_{1} \approx-\overline{\mathbf{Q}}_{1}^{H}$ and $\overline{\mathbf{P}}_{2} \approx-\frac{1}{2} \overline{\mathbf{F}} \overline{\mathbf{F}}^{H}$ are components of the noise and signal-space perturbations, with $\overline{\mathbf{Q}}_{1}$ defined on the next page.

Next, recall that $\mathbf{V}_{s} \perp \mathbf{v}_{F}$ and $\mathbf{V}_{s}^{H} \mathbf{V}_{s}=\mathbf{I}$, therefore we have $\mathbf{V}_{s}^{H} \Delta \mathbf{V}_{s}=\overline{\mathbf{P}}_{2}$.

After some manipulations based on the circular symmetry of $\Delta \mathbf{H}_{b a}$, we obtain

$$
\begin{aligned}
E\left\{\left[\mathbf{V}_{s}^{H} \Delta \mathbf{V}_{s}\right]\right\}= & -\frac{1}{2} \sigma_{F}^{2}\left(\mathbf{D} \mathbf{V}_{s}^{H} \mathbf{G} \mathbf{V}_{s} \mathbf{D}^{H}+\boldsymbol{\Sigma}_{s}^{-1} \mathbf{D}\left(\left[\sigma_{F}^{2}+\mathbf{I}+\mathbf{D}^{-1}\right]\right.\right. \\
& \left.\left.\times \mathbf{U}_{s}^{H} \mathbf{G} \mathbf{U}_{s} \mathbf{D}^{H}+\mathbf{D}^{-1} \mathbf{U}_{s}^{H} \mathbf{G} \mathbf{U}_{s}\right) \boldsymbol{\Sigma}_{s}^{-1}\right) .
\end{aligned}
$$

where the $(i, j)$ entry of $\mathbf{G}$ is

$$
\mathbf{G}_{i, j}=\sigma_{H}^{2} \operatorname{Tr}\left(\mathbf{v}_{F} \mathbf{v}_{F}^{H}\right)=\sigma_{H}^{2} .
$$

Using the second-order statistics of $\Delta \mathbf{H}_{b a}$ we obtain

$E\left\{\mathbf{E}_{s n} \mathbf{E}_{s n}^{H}\right\}=\mathbf{U}_{s}^{H} E\left[\Delta \mathbf{H}_{b a} \mathbf{V}_{n} \mathbf{V}_{n}^{H} \Delta \mathbf{H}_{b a}^{H}\right] \mathbf{U}_{s}=\mathbf{U}_{s}^{H} \mathbf{G} \mathbf{U}_{s}$

Similarly, $E\left\{\mathbf{E}_{n s}^{H} \mathbf{E}_{n s}\right\}=\mathbf{V}_{s}^{H} \mathbf{G V}_{s}$. As a result,

$$
E\left\{\mathbf{v}_{1}^{H} \Delta \mathbf{v}_{1}\right\}=E\left[\mathbf{V}_{s}^{H} \Delta \mathbf{V}_{s}\right]_{1,1} .
$$




$$
\begin{aligned}
\overline{\mathbf{Q}}_{1} \approx & \mathbf{D}\left(\mathbf{E}_{s s} \mathbf{D} \boldsymbol{\Sigma}_{s} \mathbf{E}_{n s}^{H}-\sigma_{F} \mathbf{D} \mathbf{E}_{n s}^{H} \mathbf{E}_{n n}^{H}\right) \boldsymbol{\Sigma}_{n}-\mathbf{D} \mathbf{E}_{n s}^{H} \mathbf{E}_{n n}+\sigma_{F}^{2} \mathbf{D}\left(\mathbf{E}_{s s}^{H} \mathbf{D} \mathbf{E}_{s n}-\mathbf{D} \mathbf{E}_{n s}^{H} \mathbf{E}_{n n}\right) \\
& +\sigma_{F}^{2} \boldsymbol{\Sigma}_{s}^{-1} \mathbf{D}\left(\mathbf{E}_{s s} \boldsymbol{\Sigma}_{s}^{-1} \mathbf{E}_{s n}-\mathbf{D} \mathbf{E}_{s n} \boldsymbol{\Sigma}_{n}^{H} \mathbf{E}_{n n}+\sigma_{F}^{2} \mathbf{E}_{s s} \boldsymbol{\Sigma}_{s}^{-1} \mathbf{D} \mathbf{E}_{s n}\right)+\boldsymbol{\Sigma}_{s}^{-1} \mathbf{E}_{s s} \boldsymbol{\Sigma}_{s}^{-1}\left(\mathbf{E}_{s n}+\sigma_{F}^{2} \mathbf{D} \mathbf{E}_{s n}\right) \\
& -\sigma_{F} \boldsymbol{\Sigma}_{s}^{-1} \mathbf{D} \mathbf{E}_{s n} \mathbf{E}_{n n}+\sigma_{F} \boldsymbol{\Sigma}_{s}^{-1} \mathbf{E}_{s s} \mathbf{D} \mathbf{E}_{n s}^{H}+\sigma_{F} \boldsymbol{\Sigma}_{s}^{-1} \mathbf{D}\left(\sigma_{F}^{2} \mathbf{E}_{s s} \mathbf{D} \mathbf{E}_{n s}^{H}-\sigma_{F}^{2} \mathbf{D} \mathbf{E}_{s n} \mathbf{E}_{n n}^{H}-\mathbf{E}_{s n} \mathbf{E}_{n n}^{H}\right)+\overline{\mathbf{F}}
\end{aligned}
$$

Also,

$$
E\left\{\Delta \sigma_{1}\right\}=E\left\{\left[\Delta \boldsymbol{\Sigma}_{s}\right]_{1,1}\right\}
$$

where

$$
E\left\{\Delta \boldsymbol{\Sigma}_{s}\right\} \approx\left(\sigma_{F}^{2} \mathbf{U}_{s}^{H} \mathbf{G} \mathbf{U}_{s} \mathbf{D}^{H}+\mathbf{U}_{s}^{H} \mathbf{G} \mathbf{U}_{s}\right) \boldsymbol{\Sigma}_{s}^{-1}
$$

$+\boldsymbol{\Sigma}_{s} E\left[\mathbf{V}_{s}^{H} \Delta \mathbf{V}_{s}\right]-\mathbf{D}\left(\boldsymbol{\Sigma}_{s} \mathbf{V}_{s}^{H} \mathbf{G} \mathbf{V}_{s} \boldsymbol{\Sigma}_{s}+\sigma_{F}^{2} \mathbf{U}_{s}^{H} \mathbf{G} \mathbf{U}_{s}\right) \mathbf{D}^{H} \boldsymbol{\Sigma}$

and,

$$
E\left\{\Delta \sigma_{1}^{2}\right\}=\left[\mathbf{U}_{s}^{H} \mathbf{G} \mathbf{U}_{s}\right]_{1,1}
$$

Finally,

$$
E\left\{\Delta \sigma_{1}^{2}\right\} \approx E\left[\mathbf{E}_{s s} \mathbf{E}_{s s}^{H}\right]_{1,1}=\left[\mathbf{U}_{s}^{H} \mathbf{G} \mathbf{U}_{s}\right]_{1,1} .
$$

Thus, by substituting (30)-(34) into (23), the naive SINR averaged over the channel perturbation can be obtained. It is shown in Section $\mathrm{V}$ that even relatively small perturbations in CSI lead to a significant degradation in the naive SINR.

To second order, Alice's use of imperfect transmit beamformers does not impact the SINR available to Eve. As far as Eve is concerned, use of $\mathbf{v}_{1}+\Delta \mathbf{v}_{1}$ rather than $\mathbf{v}_{1}$ as the transmit beamformer for the desired signal, and $\mathbf{T}^{\prime}+\Delta \mathbf{T}^{\prime}$ rather than $\mathbf{T}^{\prime}$ as the interference precoder, has no net effect on her performance.

\section{Robust BeAmforming APPROACH}

While the instantaneous CSI perturbation cannot be determined, if Bob and Alice have information about the statistics of the perturbation, then they may take remedial measures to overcome at least some of the significant SINR degradation that occurs with the naive scheme. In particular, if Bob and Alice have knowledge of $\mathbf{C}_{\Delta \mathbf{H}_{b a}}$, then the spatial covariance of the artificial interference that impacts Bob can be calculated, and incorporated into the maximum SINR beamformer. In this section, we examine such an approach for the case where the statistics are assumed to be available to both Alice and Bob, and Alice does not possess CSI for Eve as before.

When Alice has imperfect CSI for Bob and applies a mismatched transmit beamformer, the interference-plus-noise portion of Bob's received signal is, from (3),

$$
\tilde{\mathbf{n}}_{b}=\mathbf{H}_{b a} \mathbf{z}^{\prime}+\mathbf{n}_{b},
$$

with covariance

$$
E\left\{\tilde{\mathbf{n}}_{b} \tilde{\mathbf{n}}_{b}^{H}\right\}=\mathbf{Q}_{\text {int }} .
$$

Using the second-order perturbation approach of the previous section, the effective interference-plus-noise covariance matrix $\mathbf{Q}_{\text {int }}$ can be computed as

$$
\mathbf{Q}_{i n t}=E\left\{\mathbf{H}_{b a} \mathbf{z}^{\prime} \mathbf{z}^{\prime H} \mathbf{H}_{b a}^{H}+\mathbf{n}_{b} \mathbf{n}_{b}^{H}\right\}
$$

$$
\begin{aligned}
=\tilde{\beta} & \left(\mathbf{H}_{b a} \mathbf{H}_{b a}^{H}-\sigma_{1}^{2} \mathbf{u}_{1} \mathbf{u}_{1}^{H}\right)-\beta \sigma_{1}^{2} \mathbf{u}_{1} E\left\{\Delta \mathbf{v}_{1}\right\} \mathbf{H}_{b a}^{H} \\
& +\sigma_{1} \beta \mathbf{H}_{b a} E\left\{\Delta \mathbf{v}_{1}\right\} \mathbf{u}_{1}^{H}+\sigma_{b}^{2} \mathbf{I} .
\end{aligned}
$$

The interference-plus-noise covariance matrix averaged over the CSI error statistics requires the expected value $E\left\{\Delta \mathbf{v}_{1}\right\}=$ $E\left\{\left[\Delta \mathbf{V}_{s}\right]_{:, 1}\right\}$. This can be obtained from

$$
E\left\{\Delta \mathbf{V}_{s}\right\}=\mathbf{v}_{F} E\left\{\overline{\mathbf{P}}_{1}\right\}+\mathbf{V}_{s} E\left\{\overline{\mathbf{P}}_{2}\right\} .
$$

Define $\left[\mathbf{G}^{\prime}\right]_{i, j}=\sigma_{H}^{2} \operatorname{Tr}\left(\mathbf{D}^{H}\right)$. The expected value of $\overline{\mathbf{P}}_{2}$ is given in (28). Once again, exploiting the circular symmetry of $\Delta \mathbf{H}_{b a}$ leads to the following remaining expectation terms:

$$
\begin{aligned}
E\left\{\overline{\mathbf{P}}_{1}\right\} & =\mathbf{v}_{F}^{H} \mathbf{G} \mathbf{V}_{s} \mathbf{D}^{H}\left(\mathbf{I}+\sigma_{F}^{2}\right)-\mathbf{u}_{F}^{H} \mathbf{G}^{\prime} \mathbf{U}_{s}\left(\mathbf{I}+\mathbf{D}^{H}\right) \boldsymbol{\Sigma}_{s}^{-1} \\
& +\sigma_{F} \mathbf{u}_{F}^{H} \mathbf{G} \mathbf{U}_{s} \mathbf{D}^{H}\left(\sigma_{F}^{2} \mathbf{D}^{H}+\mathbf{I}\right) \boldsymbol{\Sigma}_{s}^{-1}+\sigma_{F}^{2} \mathbf{v}_{F}^{H} \mathbf{G}^{\prime} \mathbf{V}_{s} \mathbf{D}^{H} .
\end{aligned}
$$

Next, we assume that both Alice and Bob have knowledge of the CSI error statistics, which allows Alice to take a proactive role in alleviating the SINR degradation due to imperfect CSI. If Alice is aware of the interference-plus-noise covariance matrix $\mathbf{Q}_{\text {int }}$, she can select an appropriate robust transmit beamforming vector $\mathbf{t}_{r}$ as follows. Applying $\mathbf{Q}_{i n t}^{-1 / 2}$ as a prewhitening filter to Bob's received signal, we have

$$
\mathbf{Q}_{i n t}^{-1 / 2} \mathbf{y}_{b}=\mathbf{Q}_{i n t}^{-1 / 2} \mathbf{H}_{b a} \mathbf{t}_{r} z+\mathbf{Q}_{i n t}^{-1 / 2} \tilde{\mathbf{n}}_{b} .
$$

Treating $\mathbf{H}_{\text {eff }}=\mathbf{Q}_{i n t}^{-1 / 2} \mathbf{H}_{b a}$ as Bob's effective channel matrix, a suitable choice for $\mathbf{t}_{r}$ is the right singular vector corresponding to the largest singular value of $\mathbf{H}_{e f f}$, in which case Bob chooses $\mathbf{H}_{e f f} \mathbf{t}_{r}$ as his receive beamformer. Note that in this case, Alice can guarantee that Bob will experience the desired average SINR value $S$ by setting

$$
\rho=\frac{S}{\hat{\sigma}_{1}^{2} P}
$$

where $\hat{\sigma}_{1}$ is the largest singular value of $\mathbf{H}_{e f f}$. However, due to the presence of increased interference, Alice will have to devote a larger fraction of power for Bob to achieve the desired SINR, and hence less will be available to jam Eve.

\section{SIMULATION RESULTS}

We present some examples that show the SINR performance of Bob and Eve for various array sizes, target performance levels, and array perturbations. In all simulations, the channel matrices were assumed to be composed of independent, zeromean Gaussian random variables with unit variance $\left(\gamma_{e a}^{2}=1\right)$. The channel perturbation covariance matrix is assumed to be $\mathbf{C}_{\Delta H_{b a}}=\sigma_{H}^{2} \mathbf{I}$ for various values of $\sigma_{H}^{2}$. In the simulation plots, $\sigma_{H}$ is specified in $\mathrm{dB}$ according to $20 \log _{10} \sigma_{H}$. All displayed results are calculated based on an average of 3000 
independent trials. The background noise power was assumed to be the same for both Bob and Eve: $\sigma_{b}^{2}=\sigma_{e}^{2}=1$.

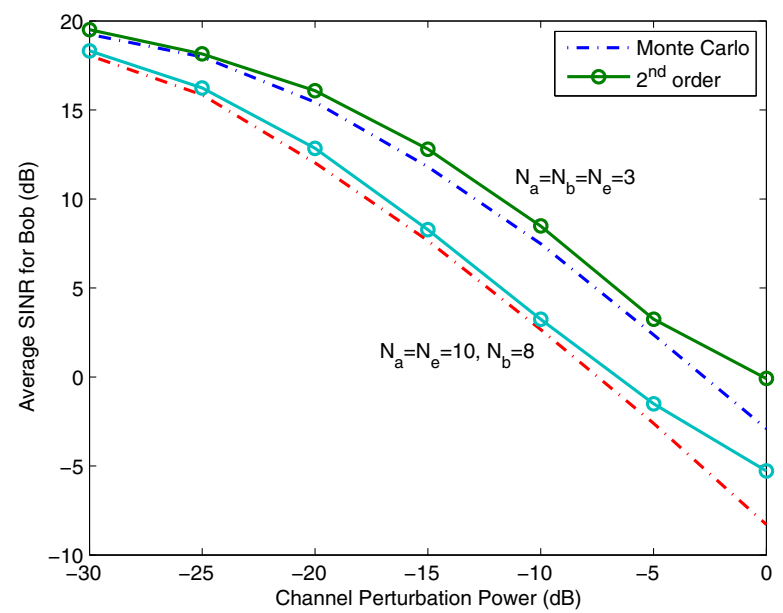

Fig. 1. A comparison of the $2^{\text {nd }}$-order naive SINR approximations with Monte Carlo SINR results for $N_{a}=N_{b}=N_{e}=3$ and $N_{a}=N_{e}=$ $10, N_{b}=8$.

In Figure 1, we compare the SINR expressions for the naive case based on second-order perturbation theory derived in Section III with measured SINR values from simulations for a range of channel perturbation powers. The set of channel matrices have dimensions of either $N_{a}=N_{b}=N_{e}=3$ or $N_{a}=N_{e}=10, N_{b}=8$, and the desired SINR for Bob is set to $S=20 \mathrm{~dB}$. For both antenna configurations, the secondorder approximations appear to be accurate even for channel perturbations of the same order as the channel coefficients themselves. Note that Bob's SINR is substantially impacted, even for relatively small values of $\sigma_{H}$. For example, when $N_{a}=10$, Bob loses $7 \mathrm{~dB}$ of SINR for the relatively small value $\sigma_{H}=0.1$.

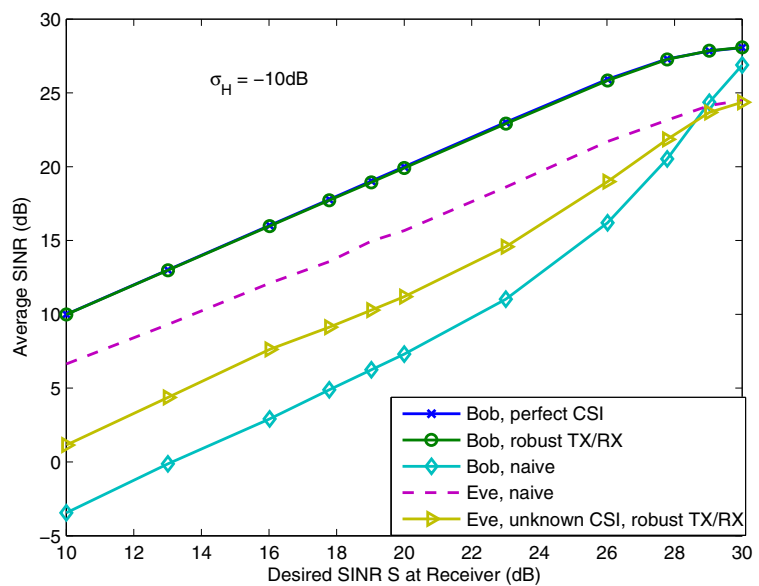

Fig. 2. Measured SINR values versus desired SINR for Bob with perfect and imperfect CSI at Alice for $N_{a}=N_{b}=N_{e}=3$ antennas, $\sigma_{H}=-10 \mathrm{~dB}$.

Figure 2 shows the resulting SINRs for Bob and Eve as a function of $S$ when the channel perturbation power is fixed at $\sigma_{H}=-10 \mathrm{~dB}$, for the case where $N_{a}=N_{b}=N_{e}=N=3$. It is evident that the naive schemes incur a significant SINR penalty for a relatively small channel perturbation, with the achieved SINR at the intended receiver being $12 \mathrm{~dB}$ below the target SINR and 8dB worse than the SINR for Eve. When Alice has access to the CSI error statistics and joint transmit/receive beamforming can be applied, the remaining $1 \mathrm{~dB}$ of SINR loss is retrieved successfully. Note that Eve's SINR also increases by about $2 \mathrm{~dB}$ with joint transmit/receive beamforming for large $S$, since Alice has been forced to increase $\rho$ and hence decrease the amount of artificial interference.

\section{CONCLUSIONS}

We conducted a detailed second-order perturbation analysis in order to precisely quantify the effects of inaccurate CSI on beamforming schemes that incorporate artificial interference. The proposed approaches rely heavily on the availability of accurate CSI, and their performance are shown to be quite sensitive to imprecise channel estimates. To reduce the impact of the CSI errors, we proposed a robust beamforming scheme that is able to recover a large fraction of the SINR lost due to the channel estimation errors. This technique was shown to perform very well for moderate CSI errors, but ultimately a large enough channel mismatch can eliminate the secrecy advantage of using artificial noise.

\section{REFERENCES}

[1] A. D. Wyner, "The wire-tap channel," The Bell System Technical Journal, vol. 54, pp. 1355-1387, 1975.

[2] S. Leung-Yan-Cheong and M. Hellman, "The gaussian wire-tap channel," IEEE Trans. Inform. Theory, vol. 24, no. 4, pp. 451-456, July 1978.

[3] I. Csiszar and J. Korner, "Broadcast channels with confidential messages," IEEE Trans. Inform. Theory, vol. 24, no. 3, pp. 339-348, May 1978.

[4] A. Khisti, G. Wornell, A. Wiesel, and Y. Eldar, "On the gaussian MIMO wiretap channel," in IEEE Intl Symp. on Info. Theory, pp. 2471-2475, June 2007.

[5] F. Oggier and B. Hassibi, "The secrecy capacity of the MIMO wiretap channel," in IEEE Intl Symp. on Info. Theory, pp. 524-528, July 2008.

[6] A. Khisti and G. W. Wornell, "Secure transmission with multiple antennas II: The MIMOME wiretap channel", submitted to IEEE Trans. Inform. Theory, Aug. 2008.

[7] T. Liu and S. Shamai, "A note on the secrecy capacity of the multiantenna wiretap channel," IEEE Trans. Inform. Theory, vol. 55, no. 6, pp. 2547-2553, June 2009 .

[8] A. L. Swindlehurst, "Fixed SINR solutions for the MIMO wiretap channel," in IEEE ICASSP, pp. 2437-2440, Apr. 2009.

[9] A. Mukherjee and A. L. Swindlehurst, "Fixed-rate power allocation strategies for enhanced secrecy in MIMO wiretap channels," Proc. of IEEE SPAWC, pp. 344-348, June 2009.

[10] S. Goel and R. Negi, "Guaranteeing secrecy using artificial noise," IEEE Trans. Wireless Commun., vol. 7, no. 6, pp. 2180-2189, June 2008.

[11] X. Zhou and M. R. McKay, "Physical layer security with artificial noise: Secrecy capacity and optimal power allocation", in Proc. Int. Conf. on Sig. Proc. and Commun. Syst., Omaha, NE, Sept. 2009.

[12] E. Tekin and A. Yener, "The general gaussian multiple-access and twoway wiretap channels: achievable rates and cooperative jamming," IEEE Trans. Inform. Theory, vol. 54, no. 6, pp. 27352751, June 2008.

[13] M. Bloch, J. Barros, M. Rodrigues, and S. W. McLaughlin, "Wireless information-theoretic security," IEEE Trans. Inform. Theory, vol. 54, no. 6, pp. 2515-2534, June 2008.

[14] M. Bloch and J. Laneman, "Information-spectrum methods for information-theoretic security," Information Theory and Applications Workshop, pp. 23-28, February 2009.

[15] Z. Xu, "Perturbation analysis for subspace decomposition with applications in subspace-based algorithms," IEEE Trans. Signal Proc.., vol. 50, no. 11, pp. 2820-2830, Nov. 2002. 